relating to living with RP, was facilitated by members of the Large Airway Collapse with Symptoms (LACS) national network.

The facilitators were experienced in running focus groups and had clinical awareness of RP to support discussion when needed. The semi-structured session explored: symptoms; effect on everyday life (emotional, physical, impact on others); diagnosis(time span, professionals involved, ongoing monitoring); healthcare professional support. The session concluded with a priority exercise where participants received four 'resource' coins to distribute as they wished to themed issues (as defined collectively by the group).

Results Twenty-two participated; 13 RP suffers and 9 carers. The group fully engaged and there were no conflicts, despite differing opinions. Despite some valued on-line peer support groups general consensus was provision is limited for those living with RP; the majority had never met another RP sufferer/carer until the session.

Group defined themed issues for RP suffers included: lack of understanding; loss of identity; breathlessness; pain; fatigue; impact on relationships; memory impedance. Carer themed issues were similar: pain; lack of appropriate support; restrictions on planning ahead; impact on social life. The allocation of resource across the groups differed in priority (Table 1 ).

Conclusion Common issues effect both RP suffers and their carers but there are variations in the hierarchy of burden. We acknowledge this is a preliminary engagement exercise. However, the key issues identified should form the foundation for further research focus groups to guide appropriate patient-centred care for RP.

\section{M11 THE IMPACT OF RESPIRATORY SPEECH AND LANGUAGE THERAPY ON PATIENTS' COUGH RELATED SYMPTOMS}

1J Haines, ${ }^{2} \mathrm{C}$ Slinger, ${ }^{2} \mathrm{~A}$ Vyas, ${ }^{3} \mathrm{~S}$ Chua, ${ }^{1} \mathrm{~S} J$ Fowler. ${ }^{1}$ North West Lung Centre, University Hospital of South Manchester, Manchester, UK; ${ }^{2}$ Lancashire Teaching Hospitals NHS Foundation Trust, Preston, UK; ${ }^{3}$ University of Manchester Medical School, Manchester, UK

\subsection{6/thoraxjnl-2016-209333.453}

Introduction Unexplained chronic cough may persist despite systematic evaluation and medical treatment of relevant comorbidities. Currently there are no effective, acceptable anti-tussive agents for the treatment of such patients and significant physical, social and psychological morbidity is described. The role of nonpharmacological treatment approaches and specifically speech and language therapy have been reported to be effective.

In our specialist tertiary airways service, all patients with unexplained chronic cough greater than 8 weeks in duration, remaining unexplained after investigation and supervised therapeutic trials, are referred for respiratory speech and language therapy (rSLT).

Aims To determine the effect of rSLT on the Leicester Cough Questionnaire (LCQ) and establish specifically whether the impact occurs across each of the described domains: physical, psychological and social.

Methods We included retrospective data from all patients with unexplained chronic cough who completed rSLT between January and June 2016, and who had LCQ data available before and after treatment.

Results Sixteeen full data sets [69\% female; median (range) age 58 (35-73) years] were available for analysis; rSLT median $=4$, (range $=3-6$ ) sessions. There was overall improvement in LCQ from median (range) 13.0 (7.0-18.0) pre to $17.4(8.0-21.0)$ post rSLT [minimal important difference (MID) 1.3; Wilcoxon's signed rank $\mathrm{p}<0.001]$. Each domain improved post rSLT: physical from $4.7(3.0-7.0)$ pre to $6.0(2.0-7.0)$ post (MID 0.2; $\mathrm{p}=0.004)$; psychological: from $4.0(1.0-6.0)$ to $6.1(3.0-7.0)$ (MID 0.8; $\mathrm{p}=0.001)$; and social from $4.0(2.0-7.0)$ to 5.7 (3.07.0 ) post (MID 0.2, p = 0.001). Individual answers to 10 of the 19 LCQ questions showed statistically significant improvements.

Conclusion These preliminary data indicate that rSLT improves cough related symptoms similarly cross all domains. Further investigation is needed to inform which aspects of patients' cough-related symptoms do/do not improve with therapy to guide treatment refinement. Specifically, closer investigation of response to individual LCQ questions may lead to improvements in therapeutic strategies.

\section{M12 TRPV1 POLYMORPHISM IN CHRONIC COUGH: NO EVIDENCE FOR AN EFFECT ON OBJECTIVE MEASUREMENTS OF COUGH}

${ }^{1}$ RD Turner, ${ }^{2} E$ Bourne, ${ }^{2} \mathrm{CA}$ Mein, ${ }^{3} \mathrm{SS}$ Birring, ${ }^{2} \mathrm{SO}$ Shaheen, ${ }^{1} \mathrm{GH}$ Bothamley. ${ }^{1}$ Homerton University Hospital NHS Foundation Trust, London, UK; ${ }^{2}$ Queen Mary University of London, London, UK; ${ }^{3}$ King's College London, London, UK

\subsection{6/thoraxjnl-2016-209333.454}

Introduction and objectives The afferent receptor TRPV1 is implicated in the cough reflex. Several single nucleotide polymorphisms (SNPs) in TRPV1 appear to be associated with symptoms of cough in the general population. ${ }^{1}$ We investigated whether such SNPs are associated with the objective measures of cough reflex sensitivity and 24-hour cough frequency (CF24) in chronic cough.

Methods Patients were recruited from a specialist cough clinic with cough $>2$ months' duration as the only or predominant symptom. Each underwent measurement of C5 (minimum concentration of capsaicin required to produce 5 coughs) and wore the Leicester Cough Monitor for 24 h. Saliva samples were taken to extract DNA and genotype six SNPs of TRPV1: rs161365, rs17706630, rs2277675, rs222741, rs150854 and rs224498. Mean values of C5 and CF24 were compared across genotypes and univariate linear regression was used to analyse the per minor allele associations with each outcome for each SNP, on the assumption their effects would be additive. ${ }^{1}$

Results 57 patients were recruited with underlying diagnoses including unexplained chronic cough $(\mathrm{n}=43)$, asthma $(\mathrm{n}=7)$ and gastro-oesophageal reflux $(n=2) .42(74 \%)$ were female, and median (IQR) age and duration of cough was 60 (54-66) years and 5 (3-12) months, respectively. Median (IQR) C5 was 7.8 (3.9-15.6) $\mu \mathrm{M} / \mathrm{L}$ and cough frequency 399 (181-651) coughs/24 h.

Genotype frequencies were as shown (Table). There was no evidence for an association between TRPV1 polymorphisms and C5 or CF24. This was the case regardless of diagnosis.

Conclusion This small study did not provide support for a large effect of TRPV1 polymorphism on cough frequency or cough reflex sensitivity in chronic cough. However, we lacked power to detect small/modest effects.

\section{REFERENCE}

1 Smit, et al. Respir Res 2012;13:26. 